\title{
Partnerships to Care for Our Patients and Communities During COVID-19
}

\author{
Anthony J. Viera, MD, MPH, Jacqueline Barnett, DHSc, MHS, Matthew Case, DO, \\ Carol Epling, MD, Brian Halstater, MD, Michelle Lyn, MBA, \\ Viviana Martinez-Bianchi, MD, Jobn Ragsdale, MD, Kenyon Railey, MD, \\ Kristen Said, MD, Gregory Sawin, MD, MPH, Hunter Spotts, MD, John Vaughn, MD, \\ Nancy Weigle, MD, and J. Lloyd Michener, MD
}

The Coronavirus disease 2019 (COVID-19) pandemic forced not only rapid changes in how clinical care and educational programs are delivered but also challenged academic medical centers (AMCs) like never before. The pandemic made clear the need to have coordinated action based on shared data and shared resources to meet the needs of patients, learners, and communities. Family medicine departments across the country have been key partners in AMCs' responses. The Duke Department of Family Medicine and Community Health (FMCH) was involved in many aspects of Duke University's and Health System's responses, including leadership contributions in delivering employee health and student health services. The pandemic also surfaced the biological and social interactions that reveal underlying socioeconomic inequalities, for which family medicine has advocated since its inception. Key to success was the department's ability to integrate "horizontally" with the broader community, thereby accelerating the institution's response to the pandemic. ( $\mathrm{J}$ Am Board Fam Med 2021;34:1003-1009.)

Keywords: Academic Medical Centers, Community Health Planning, CoVID-19, Family Medicine, Pandemics, Public Health, Socioeconomic Factors, Syndemic

\section{Introduction}

The coronavirus 2019 (COVID-19) pandemic brought unprecedented challenges to academic medical centers (AMCs). Educational programs quickly implemented virtual learning, outpatient clinics established video visits and organized respiratory care centers, and hospitals developed and instituted protocols for COVID-19 testing and personal protective equipment while ramping up the ability to provide intensive care and reorganize care delivery systems while keeping employees and students safe. Health care administrators modeled potential financial losses and planned contingencies. At the same time, AMCs partnered with public health leaders

This article was externally peer reviewed.

Submitted 2 March 2021; revised 24 May 2021; accepted 28 May 2021.

From the Department of Family Medicine and Community Health, Duke University School of Medicine, Durham, NC (AJV, JB, MC, CE, BH, ML, VM-B, JR, KR, KS, GW, HS, JV, NW, JLM).

Funding: None.

Conflict of interest: None.

Corresponding author: Anthony J. Viera, MD, MPH, Department of Family Medicine and Community Health, Duke University School of Medicine, 2200 West Main Street, Suite 400, Durham, NC 27705 (E-mail: anthony.viera@duke.edu). and community organizations to coordinate the response and address the disproportionate impact on people of color, shining additional light on health inequities. Primary care has been instrumental in responses to COVID-19. Indeed, the fournal has previously published an entire supplement on the topic. ${ }^{1}$

Although the many departments, clinical units, inpatient facilities, and health professions programs in an $\mathrm{AMC}$ have varied and separate structures, histories, and funding streams, the pandemic made clear the need to have coordinated action based on shared data and resources. Like many departments throughout the country, the Duke University Department of Family Medicine and Community Health (FMCH) was deeply involved in the institutional and community response to COVID-19. This article describes how FMCH coordinated activities across the department and participated in multiple key partnerships across the University, which was coordinated with the county and state.

\section{Response Activities}

Duke's Department of Family Medicine and Community Health responded to the pandemic through 
work performed in its 5 divisions: Family Medicine, Community Health, Student Health, Occupational and Environmental Medicine with Employee Health, and Physician Assistant Studies.

\section{Family Medicine Clinic}

Duke University Health System is comprised of Duke University Hospital, a quaternary care complex with approximately 1,000 inpatient beds, along with 2 community hospitals. The Duke School of Medicine has 100 students per class, all of whom complete a family medicine clerkship during their second year. The Family Medicine Residency currently trains 8 residents per year and has expanded recently with a rural track. The Family Medicine Center serves approximately 14,400 patients, mostly from Durham and nearby counties. In 2020, the clinic had 29,997 outpatient visits, of which 7,813 were virtual.

On March 13, 2020, a group of 30 COVID-19 exposed or symptomatic international travelers from a known COVID-19 hotspot arrived at Duke Family Medicine Clinic for evaluation. The majority of these patients tested positive for COVID-19. The clinic was prepared for this possibility due to advanced planning, including clear communications and partnerships with health system clinical leaders and careful attention to protocols. Key to the clinic's success were partnerships that allowed operations to continue while minimizing risk to clinicians.

First, in partnership with hospital leadership, a pre-existing virtual visit platform was made functional. The clinic was prioritized to triage patients. The clinic access model changed, so morning sessions provided in-person care for non-COVID-19 illness, and afternoons were reserved for COVID19 assessments. Patient access for both COVID-19 and non-COVID-19 care was maintained by adding virtual visit sessions.

Second, the need for much greater testing capacity quickly became apparent. In partnership with the hospital, Employee Health, and the county health department, a drive-through testing system was implemented in April 2020. The first testing tent was placed in the employee parking lot of the Family Medicine Clinic and staffed by its clinicians (including residents), nurses, and medical assistants. Patients and employees were screened virtually, and those who needed testing utilized the tent.

In addition to serving in the testing tent, several Family Medicine residents committed themselves to COVID-19 response. Residents started the
African American COVID-19 Taskforce (AACT), convening a broad coalition of Durham's Black leaders. Residents became involved in testing (and later, vaccination) events and led undergraduate students through learning and service activities related to the pandemic with their population health improvement projects.

\section{Student Health}

As both a clinical division within FMCH and a University program, Student Health normally operates in a world of partnerships beyond those typically seen in an AMC practice, but the COVID-19 pandemic required new partnerships, which have proved uniquely rewarding. The student population has risk factors brought to the forefront by the pandemic. At the onset, travel restrictions prevented many students from returning home, especially students studying outside of the United States. Initially, the focus of Student Health was helping students navigate health care remotely and return to campus or their home safely. As the pandemic progressed, the focus shifted to enabling them to return to campus and continue their academic work in a safe way for them and the community at large.

Congregate living in residence halls and apartments places students at increased risk for the spread of infectious illness. Student Health collaborated with research scientists, infectious disease and public health clinicians, and emergency management staff to implement a comprehensive, multitiered testing strategy: baseline testing of all students on campus arrival, ongoing surveillance testing of asymptomatic students, and testing of all symptomatic students. This testing strategy proved to be successful in mitigating outbreaks during the fall semester. ${ }^{2}$

The corollary to the testing strategy was a vigorous case management and outbreak control process. Seventeen full-time non-clinical university staff and over 120 volunteers were redeployed and trained to conduct contact tracing interviews, working over 12,000 hours during the fall semester. During that time, contact tracers identified and quarantined 819 students in the course of conducting 260 interviews. This effort was instrumental in keeping Duke University's positive rate among students at $0.13 \%$. Student Health provided clinical oversight to the contact tracing and isolation care team responsible for supporting students requiring quarantine and isolation housing. 
The need to continue providing non-COVID-19 clinical care for students in the face of overwhelming staffing demands required additional partnerships across the health system. Departmental and health system collaborations facilitated testing and respiratory care for students at designated urgent respiratory care centers outside of Student Health. This collaboration, along with partnerships with colleagues in information technology (IT), legal, and compliance offices, allowed Student Health to increase telemedicine offerings for students, thereby maintaining access to care.

The decision to bring over 13,000 students back to campus in fall 2020 during the pandemic was fraught with risk. Fears of straining local health care resources and possibly endangering the health of vulnerable community members had to be weighed against the genuine impact that loss of the largest economic driver in the region might have on those same community members' ability to maintain basic levels of financial, social and health support. Student Health leadership worked with senior institutional leadership, scientists, clinicians, and governmental leaders to effectively and safely meet the needs of all constituents, allowing students to return with aggressive testing and tracing plans in place.

\section{Duke Physician Assistant Program and Medical Students as Contact Tracers}

The Duke Physician Assistant (PA) Program students were removed from all clinical rotations effective March 17, 2020. In the following weeks, the Duke PA Program worked proactively with the Durham County Department of Public Health to create a novel "Contact Tracing Public Health Elective."

The PA Program leadership advocated for the rotation, noting that students could fill a dire community need, aligning the social justice mission of the PA Program while providing an excellent public health partnership experience to support the Durham community. Students were supported by appropriate PPE, social distancing, and safety protocols to maintain safety for this elective rotation.

To date, 15 Duke PA students participated in the Contact Tracing community health elective, each working approximately 30 hours per week conducting $10-15$ contact calls per day. These efforts provided critical bandwidth for the health department COVID-19 efforts while simulta- neously expanding the students' perspective and knowledge of factors related to social determinants of health, community resources, and the critical work of the health departments. Students noted that the best parts of the course included working with public health employees, speaking with diverse community members, and learning more about the health department.

\section{Occupational and Environmental Medicine}

Occupational and Environmental Medicine (OEM) is another division within FMCH, and within OEM sits Duke Employee Occupational Health and Wellness (EOHW). EOHW provides health services for over 42,000 faculty and staff of Duke University and Duke University Health System. Due to its role on the Duke Emergency Management Steering Committee, EOHW began responding to COVID19 in January 2020. EOHW was part of the team of University partners advising the faculty, staff, and students based at Duke Kunshan University in China and a large number of faculty and students who traveled between China and Durham. That University Emergency Response team had to pivot to address COVID-19 locally quickly.

The first week of March brought the everincreasing stream of international travelers returning to campus. EOHW worked to track these returning faculty and staff and develop protocols for symptom monitoring, quarantining, and testing. Infectious Disease colleagues assisted with developing protocols, and the University's Emergency Services assisted with logistics of where returning faculty, staff, and students could quarantine and ensure that they had access to essential items, including food and a thermometer.

The second week of March brought the first positive employee cases. In those early days, policy development, quarantining, answering phone and e-mail questions, contact tracing, and positive case management were accomplished by 2 physicians and 2 senior occupational health nurses. Quickly overwhelmed by the recordkeeping, EOHW leadership recalled their Occupational and Environmental Medicine fellow from his away rotation. In a matter of days, he developed an electronic recordkeeping platform to serve as a complete electronic record for everything related to employee COVID-19 concerns. This platform houses all data related to symptom monitoring, testing, exposure assessments, case management, and work status. Student Health later adopted it and 
continues to be updated and refined, most recently adding capacity to track COVID-19 vaccinations.

Beginning in January 2020, EOHW leaders served on the Duke Health System Command Center with representatives from the Health System formulating a united response to the pandemic's rapidly changing realities. Discussions were held with laboratory leadership and Infection Prevention to determine parameters for using the very limited number of tests on the most appropriate employees. EOHW was also able to partner with IT colleagues in the Health System to coordinate extracting testing data from the hospital's EMR. The separate IT department for the University also played a vital role in fine-tuning the platform. Both IT departments made symptom monitoring apps customized for the different populations. It became clear that it was vital for the departments to communicate with employee health and each other.

To assist with the ever-increasing workload, the Duke Graduate Medical Education leaders sent out a call for any available trainees to assist EOHW and worked to develop approved rotations for them. Residents from Anesthesia, Pathology, Family Medicine, Medicine/Pediatrics, and Medicine/ Psychiatry rotated to EOHW to assist with contact tracing, answering employee phone calls, and ordering tests. They were also vital during policy and protocol development for contact tracing to provide feedback in real-time.

Duke Nursing provided many redeployed nurses, and Health System Operations assisted in setting up an Employee COVID-19 hotline to manage incoming inquiries. EOHW also collaborated with clinical operations leaders, the Duke physician practice, and nursing leadership to establish multiple drive-through testing tents to ensure capacity for employees. Redeployed nurses and providers from throughout the Health System assumed the role of case managers to monitor and support employees who sustained COVID-19 infection. Collaboration with colleagues in the Duke Family Medicine Clinic ensured symptomatic employees had access to testing in the early days and video or in-person visits as indicated by their symptoms and health status.

To establish a contact tracing team, all employees in Duke's Employee Wellness program were redeployed to serve in this role. The contact tracing procedures and protocols were developed by the
EOHW leadership team, which also trained the staff (wellness nurses, nutritionists, athletic trainers, and administrative staff) on how to move through the contact tracing process. A 1-week rotation was created to provide $\mathrm{PA}$, and medical students experience in contact tracing during their Family Medicine rotation. Biomedical Sciences Masters students rotated regularly with the EOHW COVID-19 Population Health Service. Partnerships with the local Health Departments and the VA health system are also ongoing.

EOHW is also co-leader of the Duke COVID19 Vaccination Steering Committee. Working with IT and the Occupational and Environmental Safety Office, the electronic platform for influenza vaccinations was adapted to capture COVID-19 vaccinations and communicate with employees regarding the timing of second vaccine doses. At the request of the NCDHHS, the Health System Operations team rapidly expanded employee vaccination clinics and is currently implementing high throughput patient vaccination clinics.

\section{Anti-Racism Efforts}

COVID-19 is not just a pandemic, but a syndemic, a global infectious disease outbreak with biological and social interactions that reveals underlying socioeconomic inequalities and requires attention to these inequities for containment. ${ }^{3}$ One of these underlying inequities is systemic racism. As a Department founded in 1966 to train clinicians "...who will bridge the gap between the advances being made at the research level of medical sciences and their application at the family and community level," the increased concerns about racism found deep resonance. ${ }^{4}$ The well-publicized police killings of 2020 fueled a renewed anti-racism movement across the institution, and the department played a significant role in framing and assisting the institution with leadership in this work.

The Duke PA Program was a leader in the early engagement of students, staff, and faculty wrestling with the pandemic of systemic racism. Within the School of Medicine, the PA Program was the first to publish an anti-racism statement and share it broadly. One project that emerged from these efforts was a video blog that gained national recognition giving voice to students struggling to reconcile their roles, learning, and lived experiences in the face of police killings of George Floyd, Breonna Taylor, and Ahmaud Arbery. ${ }^{5}$ Both Black and White students 
stepped forward to share struggles of oppression and their journeys to become authentic allies.

Educational programming evolved to address students' concerns with systemic racism and the flashpoint of police killings of unarmed Black citizens. The school's Student National Medical Association chapter wrote a 13-page letter to School of Medicine administration sharing concerns and demanding action. This letter included 8 pages of students sharing their painful lived experiences as students training at Duke. In partnership with the School, department educational program leaders continue to work to address concerns stemming from systemic racism.

Two longitudinal courses within the MD program's curriculum are led by members of the Department, the Clinical Skills Foundation (CSF) course and the Cultural Determinants of Health $\&$ Health Disparities Course (CDHD). These are required yearlong classes for first-year students. At the direction of the FMCH Course Director, the CSF course abandoned traditional didactics to create space for students to share and reflect on racism. The CDHD Course, tasked with exploring health disparities and the impact of socio-cultural influences on a broad spectrum of outcomes, also highlighted recent sociopolitical touchpoints through 2 sessions specifically focused on race/ ethnicity.

The PA Program shifted the focus of an entire 2-semester course from 1 of individual patient care experiences to a broader focus on structural and socio-cultural influences on health and well-being. The course now explores structural competencies; population health; poverty, housing, health; disability and ableism; race, racism, racial/ethnic disparities; sexual and gender minority health; medical ethics; and issues of access and equity.

The Department continues to coordinate efforts around Diversity, Equity, Inclusion (DEI), and antiracism across its divisions and multiple educational programs while aligning with broader AMC and University endeavors. In the Duke University Moments to Movement campaign, 4 committees explore anti-racism within the School of Medicine, 1 each for staff, faculty, health profession students, and graduate students/ postdocs. FMCH department members are cochairs on 2 of those committees. In November 2020, DEI leaders in the department started meeting monthly with the goal to coordinate efforts, bridge silos, and ultimately craft a comprehensive DEI/anti-racism strategy for the department.
The department also decided to add a DEI goal to its faculty annual review process to systematically begin proactive conversations in this critical sphere with all faculty. The aggregated responses are informing next steps in strategic planning.

\section{Health Equity and Community Advocacy}

The department's Director for Health Equity coled the founding of a multi-stakeholder partnership, The Latinx Advocacy Team and Interdisciplinary Network for COVID-19 (LATIN-19). LATIN-19 started meeting via Zoom on March 18, with a few members representing Duke Health, the local Health Department, City of Durham and Durham Public Schools, and a few local community organizations to plan a pandemic response that would meet the needs of the local Latinx community. The interest grew to more than 700 members representing multiple organizations and partnerships. At the meetings, community members share stories with decision-makers in a safe space, participants examine real-time data, and ideas for addressing the needs of the Latinx community are discussed.

Through its multisector partnership, LATIN-19 has promoted scientific and health equity, rendering it a trusted partner in the community and a platform to share problems and bring solutions. The virtual platform facilitated broad and robust participation. One of the critical successes of the group has been the establishment of trust with the Latinx community. This trust has been key in mitigating the disparate impact of COVID-19 on this group. LATIN-19 is committed to working beyond the pandemic to improve Latinx health and quality of life. Several months into the pandemic, our Director for Health Equity was called to serve as a consultant with the North Carolina Department of Health and Human Services (NCDHHS) to help shape the pandemic response for Latinx communities and other marginalized communities of color.

To stem the rising rates of COVID-19 infections in Durham's historically marginalized communities, the Community Health Division set up a testing site in the parking lot of 1 of its long-standing neighborhood clinics. The site connects to contact tracers at the Durham County Health Department to offer immediate testing to those exposed. While only open 2 evenings a week and Saturday mornings, the site tested more than 4000 individuals, with $38 \%$ identified as Black/African 
American and $40 \%$ as Hispanic/Latinx. The average positivity rate from July 2020 until the end of February 2021 was $20.5 \%$, almost double the state's highest average. In addition to testing, the site provides food and supplies as well as linkages to community resources.

With its multi-decade-long relationships in Durham and several surrounding counties, the Division was uniquely and expertly positioned to work with NCDHHS to provide social support services for those who had to isolate due to COVID-19. The initiative sought to reach historically marginalized populations in hardest-hit counties. In less than 2 weeks, the Division received commitments from a network of community-based organizations to deliver food boxes, supplies, private transportation, or prepared meals to individuals and families in 3 counties for reimbursement rates determined by the state. In addition, the Division processed relief payments to assist individuals/families in meeting basic needs. Within the first few weeks of operation, the program was expanded to serve 4 additional counties. Within 6 months, the program served more than 6000 households, with $79 \%$ identifying as Black/African American and $17 \%$ as Hispanic/Latinx. The initiative provided more than $\$ 2.5$ million in relief payments, 12,500 food boxes, 61,000 meals, and 10,500 COVID-19 supply kits.

\section{Discussion}

The COVID-19 syndemic has driven rapid, shortterm changes in health care delivery, including new and stronger partnerships within clinical systems, while also focusing attention and accelerating broader partnerships that address its deeper roots. The immediate responses demonstrated an enhanced degree of health system interdependency and integration of efforts, as no 1 clinical nor academic unit could respond effectively on its own. Whether it was shifting clinical care to telehealth, student rotations to include contact tracing, or the engagement of student and employee health programs in aggressive testing and contact tracing, each unit required and received support from others across the institution, as each quickly adapted to meet the needs of those they serve. Common to these efforts has been consistent, fast communication about needs and potential solutions, and the ability to rally problem-solving teams quickly. While these teams and partnerships (Table 1) developed rapidly, longer-term collaborations are likely and may serve as the basis for deeper changes.

The syndemic's profound effects lie outside the health system, found in "new" awareness of the systemic effects of racism on health (and the availability of health care services), as well as of the inability of clinical systems to directly address the

Table 1. Overview of Department Response Activities and Partnerships

\begin{tabular}{|c|c|}
\hline Response Activity/Group & Examples of Key Partnerships \\
\hline Family Medicine Clinic and Residency & $\begin{array}{l}\text { Hospital system leadership, nursing leadership, Student Health, } \\
\text { Employee Health, County Health Department }\end{array}$ \\
\hline Student Health & $\begin{array}{l}\text { University leadership, Hospital system leadership, School of } \\
\text { Medicine leadership, Infectious Diseases, Hospital } \\
\text { Epidemiology }\end{array}$ \\
\hline Physician Assistant Program and Medical Student Clerkship & School of Medicine leadership, County Health Department \\
\hline Employee Health & $\begin{array}{l}\text { University leadership, Hospital system leadership, School of } \\
\text { Medicine leadership, Infectious Diseases }\end{array}$ \\
\hline Community Health Testing Sites and Support Services & $\begin{array}{l}\text { County Health Department, NC Department of Health and } \\
\text { Human Services, Health system leadership }\end{array}$ \\
\hline LATIN-19 & $\begin{array}{l}\text { School of Medicine Faculty from many clinical departments, } \\
\text { School of Nursing Faculty, Advanced Practice Providers } \\
\text { (midwives, nurse practitioners, physician assistants), } \\
\text { psychologists, social workers, administrators, faith-based } \\
\text { leaders, participants from other nearby health systems, public } \\
\text { school leaders, Health Department, NC Department of Health } \\
\text { and Human Services representatives, NC Medicaid } \\
\text { representative, Elected officials of the city of Durham }\end{array}$ \\
\hline Anti-Racism Efforts & $\begin{array}{l}\text { University leadership, School of Medicine leadership, Health } \\
\text { system leadership }\end{array}$ \\
\hline
\end{tabular}

LATIN-19, Latinx Advocacy Team and Interdisciplinary Network for COVID-19; NC, North Carolina. 
cascading effects of inadequate housing, food insecurity, neighborhood, and interpersonal violence. However, COVID-19 also revealed the power of community partnerships, often including health care groups, as effective change agents in advocating for needed testing, protective equipment, as well as food and housing. The examples of LATIN-19, AACT, and other community health initiatives in meeting the broader needs are powerful demonstrations of these partnerships. Department and health center efforts also do not need to be constrained to the local community. Within North Carolina and within Duke, COVID-19 accelerated the state-wide rollout of NCCare360. This state-wide platform rapidly expanded to all NC counties and now supports over 4000 community organizations, as well as both Duke and the University of North Carolina. COVID-19 should leave no doubt that health systems must be connected with organizations in the community to effectively respond to illnesses that have their roots in the community.

The successes departments and institutions have had to work in collaboration with communities can serve as guiding principles for responding to a crisis riddled with uncertainty. In response to the COVID-19 syndemic, the department could integrate "horizontally" with the broader community to help meet the needs as they unfolded. Creating and sustaining such strategic partnerships is pivotal, and AMCs must ensure processes and pathways are designed to facilitate accountability to the communities they serve.

The writing group would like to thank the entire Duke Department of Family Medicine and Community Health, including all our staff, our students and trainees, and the faculty, for their tireless dedication to serving our patients, learners, communities, and each other in pursuit of better health for all.

To see this article online, please go to: http://jabfm.org/content/ 34/5/1003.full.

\section{References}

1. DeVoe J, Bazemore A. Primary care in the COVID19 pandemic: essential, and inspiring. J Am Board Fam Med 2021;34:S1-S6.

2. Denny TN, Andrews L, Bonsignori $M$, et al. Implementation of a pooled surveillance testing program for asymptomatic SARS-CoV-2 infections on a college campus - Duke University, Durham, North Carolina, August 2-October 11, 2020. MMWR Morb Mortal Wkly Rep 2020;69:1743-7.

3. Horton R. Offline: COVID-19 is not a pandemic. Lancet 2020;396:874.

4. Duke University Department of Family Medicine and Community Health. CFM 50th Anniversary: Origins of the Department. Available at: https:// fmch.duke.edu/all-divisions/news-and-events/cfm-50thanniversary-origins-department. Accessed August 2, 2021.

5. Duke University Department of Family Medicine and Community Health. Duke Physician Assistant Program. Duke Physician Assistant Program Addresses Racial Injustices. Available at: https://fmch.duke.edu/dukephysician-assistant-program/student-life/diversity-andinclusion. Accessed May 24, 2021. 